\title{
Inhibition of Fatty Acid Oxidation Modulates Immunosuppressive Functions of Myeloid-Derived Suppressor Cells and Enhances Cancer Therapies
}

\author{
Fokhrul Hossain $^{1,2, \dagger}$, Amir A. Al-Khami ${ }^{1, \dagger}$, Dorota Wyczechowska ${ }^{1}$, Claudia Hernandez ${ }^{1}$, \\ Liqin Zheng ${ }^{1}$, Krzystoff Reiss ${ }^{1,5}$, Luis Del Valle ${ }^{1,6}$, Jimena Trillo-Tinoco ${ }^{1}$, Tomasz Maj ${ }^{7}$, \\ Weiping Zou ${ }^{7}$, Paulo C. Rodriguez ${ }^{1,3}$, and Augusto C. Ochoa ${ }^{1,4}$ \\ ${ }^{1}$ Stanley S. Scott Cancer Center, Louisiana State University Health Sciences Center, New \\ Orleans, LA \\ ${ }^{2}$ Department of Biochemistry and Molecular Biology, Louisiana State University Health Sciences \\ Center, New Orleans, LA, 70112 \\ ${ }^{3}$ Department of Microbiology, Immunology and Parasitology, Louisiana State University Health \\ Sciences Center, New Orleans, LA, 70112 \\ ${ }^{4}$ Department of Pediatrics, Louisiana State University Health Sciences Center, New Orleans, LA, \\ 70112 \\ ${ }^{5}$ Department of Internal Medicine, Louisiana State University Health Sciences Center, New \\ Orleans, LA, 70112 \\ ${ }^{6}$ Department of Pathology, Louisiana State University Health Sciences Center, New Orleans, LA, \\ 70112 \\ ${ }^{7}$ Department of Surgery, University of Michigan
}

\begin{abstract}
Myeloid-derived suppressor cells (MDSC) promote tumor growth by inhibiting T-cell immunity and promoting malignant cell proliferation and migration. The therapeutic potential of blocking MDSCs in tumors has been limited by their heterogeneity, plasticity, and resistance to various chemotherapy agents. Recent studies have highlighted the role of energy metabolic pathways in the differentiation and function of immune cells; however, the metabolic characteristics regulating MDSCs remain unclear. We aimed to determine the energy metabolic pathway(s) used by MDSCs, establish its impact on their immunosuppressive function, and test whether its inhibition blocks MDSCs and enhances antitumor therapies. Using several murine tumor models, we found that tumor-infiltrating MDSCs (T-MDSC) increased fatty acid uptake and activated fatty acid oxidation (FAO). This was accompanied by an increased mitochondrial mass, upregulation of key FAO enzymes, and increased oxygen consumption rate. Pharmacologic inhibition of FAO blocked
\end{abstract}

Correspondence should be addressed to Augusto C. Ochoa, MD. Stanley S. Scott Cancer Center, Louisiana Cancer Research Consortium (LCRC), Louisiana State University Health Sciences Center. 1700 Tulane Ave Room 910, New Orleans, LA, 70112, USA, Tel: (504) 210-2828; aochoa@1suhsc.edu.

† Authors contributed equally to this work.

Competing interests: The authors do not have any financial interests or conflict of interest to disclose 
immune inhibitory pathways and functions in T-MDSCs and decreased their production of inhibitory cytokines. FAO inhibition alone significantly delayed tumor growth in a T celldependent manner and enhanced the antitumor effect of adoptive T-cell therapy. Furthermore, FAO inhibition combined with low-dose chemotherapy completely inhibited T-MDSCs immunosuppressive effects and induced a significant antitumor effect. Interestingly, a similar increase in fatty acid uptake and expression of FAO-related enzymes was found in human MDSCs in peripheral blood and tumors. These results support the possibility of testing FAO inhibition as a novel approach to block MDSCs and enhance various cancer therapies.

\section{Introduction}

Stromal cells in the tumor microenvironment promote tumor growth and metastatic spread, limit the antitumor response to immunotherapy, and protect tumors from the effect of chemotherapy and radiation therapy (1-3). Prominent in the tumor microenvironment are tumor-infiltrating myeloid-derived suppressor cells (T-MDSC) that, in addition to blocking $\mathrm{T}$-cell function and protecting tumors from the effect of chemotherapy and radiation therapy, support the expansion of regulatory T cells (Treg) $(4,5)$, further enhancing this highly immunosuppressive microenvironment. T-MDSCs use several mechanisms to block T-cell function, including the depletion of L-arginine by arginase I, the induction of T-cell apoptosis by nitric oxide (NO), and the synthesis of peroxynitrite (PNT) (6-8). The plasticity of MDSCs and the redundancy of these mechanisms have been shown by the fact that blocking one specific immunosuppressive mechanism induces the upregulation of the remaining pathways and only results in a partial recovery of T-cell function. Thus, therapies aimed at inhibiting MDSCs have been limited to myelosuppressive chemotherapeutic agents (gemcitabine and 5-fluoruracil) and multi-tyrosine kinase inhibitors (sunitinib) $(9,10)$. Therefore, better approaches to inhibit MDSCs and enhance cancer therapies, in particular cancer immunotherapy, are needed.

The last decade has seen major progress in understanding the energy metabolic pathways used by different immune cell subpopulations (11-14). Effector T cells are highly glycolytic, while Tregs and memory $\mathrm{T}$ cells use fatty acid oxidation (FAO) (15-17). Similarly, M1 macrophages and granulocytes preferentially use glycolysis (18), while M2 macrophages rely on FAO $(11,19,20)$. In the present study, we aimed to characterize the energy metabolic pathway(s) used by T-MDSCs, establish its impact on the immunosuppressive mechanisms, and test whether inhibition of this pathway would block MDSCs and enhance antitumor therapies. The results showed that, upon infiltrating the tumor, MDSCs increased the incorporation of fatty acids and activated FAO. This was accompanied by an increased mitochondrial biogenesis, upregulation of key FAO enzymes, and increased oxygen consumption rate (OCR). Inhibition of FAO blocked the tolerogenic function and immunosuppressive mechanisms of T-MDSCs and resulted in a T celldependent inhibition of tumor growth. More importantly, FAO inhibition enhanced the antitumor effect of low-dose chemotherapy and adoptive cellular therapy (ACT). Therefore, targeting FAO represents a novel approach to globally inhibiting the function of T-MDSCs and enhancing the antitumor effect of various cancer therapies. 


\section{Materials and Methods}

\section{Human Peripheral Blood Cells}

Samples were obtained from consented patients and donors under approved IRB protocols. PBMCs were separated on Ficoll-Paque Plus (GE Healthcare Life Sciences). MDSCs $\left(\mathrm{CD} 14^{\text {neg }} \mathrm{CD} 33^{+} \mathrm{HLA}^{-D R^{\text {neg }}} \mathrm{CD} \mathrm{Cb}^{+}\right.$) were sorted using a BD FACSAria (BD Biosciences, San Jose, CA). Polymorphonuclear granulocytes (PMN) were isolated by suspension over 3\% dextran, as described (21).

\section{Mouse strains, cell lines, and therapeutic models}

C57BL/6 mice ( 8 - 10 week old female) were purchased from Harlan laboratories (Indianapolis, IN), and OT-1 TCR transgenic mice were from The Jackson Laboratory (Bar Harbor, ME). Lewis lung carcinoma (3LL) and MCA-38 colon adenocarcinoma (American Type Culture Collection, Manassas, VA) were cultured in RPMI 1640 (Lonza-Biowhittaker, Walkerville, MD) supplemented with 10\% fetal bovine serum (Hyclone, Logan, UT), 25 mM HEPES, $4 \mathrm{mM}$ L-glutamine, and $100 \mathrm{U} / \mathrm{ml}$ penicillin, streptomycin (all from Life Technologies, Grand Island, NY). 3LL cells expressing Ovalbumin (3LL-OVA) were generated, as we previously described (22). 3LL and MCA-38 cells were periodically tested and validated to be mycoplasma-free.

In vivo tumor models were generated by injecting C57BL/6 mice s.c. with $1 \times 10^{6} 3 \mathrm{LL}$ cells or $2.5 \times 10^{5} \mathrm{MCA}-38$ cells, followed by daily i.p. injections of $50 \mathrm{mg} / \mathrm{Kg}$ of the FAO inhibitors etomoxir or ranolazine (Sigma-Aldrich). For depletion of T-cell subsets, mice were injected i.p. with $500 \mu \mathrm{g} /$ mouse anti-CD4 (GK1.5) or anti-CD8 (2.43) (BioXCell, West Lebanon, $\mathrm{NH}) 1$ day before and 2 days after tumor injection, followed by injection of 250 $\mu \mathrm{g} /$ mouse every 5 days throughout the experiment. To test the synergy between FAO inhibition and low-dose chemotherapy, 3LL or MCA-38 tumor-bearing mice were treated daily for 6 days with etomoxir, starting on day 1 after tumor injection, followed by a single i.p. dose of cyclophosphamide (CTX; Sigma-Aldrich) at $200 \mathrm{mg} / \mathrm{Kg}$ on day 7. To test the effect on established tumors, tumor cells were allowed to grow for 5 days, followed by etomoxir injections on days 6 through 10 (4 days) and a single injection of CTX (200 $\mathrm{mg} / \mathrm{Kg}$ ) on day 11 . To determine the effect of FAO inhibition on ACT, mice were injected s.c. with $1 \times 10^{6} 3 \mathrm{LL}-\mathrm{OVA}$ cells and treated with etomoxir daily throughout the experiment. OT-1 T cells $\left(2.5 \times 10^{6}\right)$ were adoptively transferred on day 14 , and mice were vaccinated with $100 \mu \mathrm{g}$ /mouse SIINFEKL peptide (American Peptide Company, Sunnyvale, CA) on day 15 after tumor injection. Ten days after the transfer, spleens were recovered and challenged with SIINFEKL for 24 hours, after which they were monitored for IFN $\gamma$ production by Elispot ( $\mathrm{R} \& \mathrm{D}$ systems). Activated OT-1 T cells were generated by stimulating splenocytes from OT-1 TCR transgenic mice in complete media with SIINFEKL peptide $(1 \mu \mathrm{g} / \mathrm{ml})$ and IL2 $(100 \mathrm{U} / \mathrm{ml})$ for 3 days. Tumor volume was measured using calipers and calculated using the formula: [(small diameter $)^{2} \times($ large diameter $) \times 0.5$ ] Experiments using animals were approved by the LSU-IACUC. 


\section{Isolation of T cells and MDSCs}

$\mathrm{CD}^{+} \mathrm{T}$ cells were isolated from spleens of C57BL/6 mice using a $\mathrm{T}$ cell negative isolation kit (Life Technologies). Purity exceeded 95\%. For T-MDSCs, tumors were digested with DNAse and Liberase (Roche USA, Branchburg, NJ) at $37^{\circ} \mathrm{C}$ for 1 hour, and T-MDSCs were isolated from tumor single-cell suspensions, as described (23). Similarly, splenic MDSCs were isolated from spleens of tumor-bearing mice, and normal myeloid cells (nMC) were isolated from spleens of control mice. The purity of different MDSC preparations ranged from 90 to $99 \%$. $\mathrm{Ly}_{6 \mathrm{C}^{+}}$and $\mathrm{Ly} 6 \mathrm{G}^{+}$MDSC subsets were isolated by flow cytometric cell sorting. For the generation of bone marrow-derived MDSCs (BM-MDSC), bone marrow cells were harvested from femurs and tibias of control mice and cultured with G-CSF (100 $\mathrm{ng} / \mathrm{ml})$, GM-CSF (20 ng/ml), and IL13 (80 ng/ml), as previously described (24). Cytokines were purchased from R\&D Systems (Minneapolis, MN). When indicated, etomoxir (100 $\mu \mathrm{M})$ was added on day 2 of culture.

\section{MDSC suppression of T cells}

$\mathrm{CD}^{+} \mathrm{T}$ cells were labeled with $1 \mu \mathrm{M}$ CFSE (Molecular Probes, Life Technologies) and were co-cultured with BM-MDSCs or T-MDSCs at a 4:1 T cell:MDSC ratio, in the presence of plate-bound anti-CD3 (145-2C11) and anti-CD28 (37.51) $(1 \mu \mathrm{g} / \mathrm{ml}$ each; BD

Biosciences). T-cell proliferation was measured after 72 hours by CFSE dilution. When indicated, IFN $\gamma$ production was assessed by ELISA (Biolegend, San Diego, CA).

\section{Flow cytometry}

Anti-human antibodies used to characterize cell subpopulations were: anti-CD33 (VM53), anti-HLA-DR (G46-6), anti-CD66b (80H3) (Beckman Coulter, Miami, FL), and anti-CD14 (61D3) (eBioscience, San Diego, CA). Mouse antibodies specific for CD11b (M1/70), Gr1 (RB6-8C5), Ly6C (AL-21), Ly6G (1A8), CD8 (53-6.7), and CD45.1 (A20) were obtained from BD Biosciences. Live/Dead stain kit was from Molecular Probes (Life Technologies). A Gallios flow cytometer (Beckman Coulter) was used for flow cytometry acquisition. Samples were analyzed with FlowJo software (TreeStar, Ashland, OR).

\section{Extracellular flux analysis}

Oxygen consumption rate (OCR) and extracellular acidification rate (ECAR) were measured using XF-24 and XFe-24 Extracellular Flux Analyzers, respectively (Seahorse Bioscience, North Billerica, MA) following manufacturer's instructions. OCR was measured in XF media containing $11 \mathrm{mM}$ glucose and $1 \mathrm{mM}$ sodium pyruvate under basal conditions and in response to $1 \mu \mathrm{M}$ oligomycin, $1 \mu \mathrm{M}$ carbonyl cyanide p-trifluoromethoxyphenylhydrazone (FCCP), and $0.1 \mu \mathrm{M}$ rotenone plus $0.1 \mu \mathrm{M}$ antimycin A. ECAR was measured in XF media containing $2 \mathrm{mM} \mathrm{L}$-glutamine under basal conditions and in response to $10 \mathrm{mM}$ glucose, 1 $\mu \mathrm{M}$ oligomycin, and $100 \mathrm{mM}$ 2-Deoxy-D-glucose (2DG).

\section{Glucose and fatty acid uptake assays}

Glucose uptake was measured using a flow cytometry-based assay, in which single-cell suspensions were incubated with $100 \mu \mathrm{M}$ fluorescent 2-(N-[7-nitrobenz-2-oxa-1,3-diazol-4yl]amino)-2-deoxyglucose (2NBDG) for 2 hours, followed by staining with different cell 
surface markers (16). Fatty acid uptake in MDSCs was determined by a fluorometric fatty acid uptake kit (Abcam, Cambridge, MA). Cells were serum-deprived for 1 hour at $37^{\circ} \mathrm{C}$ and incubated with fatty acid mixture for 30 minutes; the fluorescence signal was measured in a microplate reader.

\section{Measurement of mitochondrial mass and production of superoxide, reactive oxygen species, nitrite, peroxynitrite, and ATP}

Staining with Mitotracker green was used to determine mitochondrial mass, Mitosox red to determine mitochondrial superoxide, and 2',7'-Dichlorofluorescein Diacetate (DCFDA) to determine cellular reactive oxygen species (ROS) (Molecular Probes, Life Technologies). Nitrite production was evaluated using standard Griess reagent (Molecular Probes, Life Technologies). PNT production was assessed by quantifying nitrotyrosine residues from cell lysates by ELISA (Millipore, Billerica, MA). The levels of ATP were quantified using a bioluminescence assay kit (Molecular Probes, Life Technologies).

\section{Real-time PCR}

Total RNA was isolated using RNAeasy Mini Kit (Qiagen, Valencia, CA). cDNA was generated using iScript cDNA Synthesis Kit (Bio-Rad, Hercules, CA). Real-time PCR was performed for carnitine palmitoyltransferase 1 (CPT1), Acyl CoA dehydrogenase (ACADM), peroxisome proliferator-activated receptor gamma coactivator 1-beta (PGC1 $\beta$ ), and 3-hydroxyacyl-CoA dehydrogenase (HADHA) with Taqman primers from Applied Biosystems. Gene expression was calculated relative to $18 \mathrm{~s}$ rRNA using 2- $\Delta \Delta \mathrm{CT}$ method.

\section{Western blot}

Western blot analysis was performed using standard protocols. Proteins were electrophoresed in $8 \%$ TrisGlycine gels, transferred to polyvinylidene difluoride membranes, and immunoblotted with antibodies against arginase I (19; BD Biosciences) and $\beta$-actin (AC-74; Sigma).

\section{Bio-Plex cytokine assays and CPT1 Enzyme Activity}

Cytokines and chemokines were assessed in cell lysates using a Bio-Plex immunoassay (Bio-Rad Laboratories). CPT1 activity was measured in cell lysates based on the release of CoA-SH from palmitoyl CoA using thiol reagent 5,5-dithio-bis 2-nitrobenzoic acid (DTNB; Sigma) (25).

\section{Cancer Stem Cell Sphere assay and biomarkers}

Mouse cancer cells (3LL and MC38) were cultured for 7 days in ultra-low attachment polystyrene 6-well plates (Corning, \#3471). The cells from nearly confluent regular cell culture were washed twice in PBS and resuspended in X-Vivo 20 serum-free medium (Lonza, \#04-448Q). The cells were seated in $3 \mathrm{ml}$ of X-Vivo 20 medium 2.0×103 (MC38 cells) or $1 \times 103$ (3LL cells) with or without ETX $(100 \mu \mathrm{M})$. After 7 days spheres were counted with phase contrast microscope, with exclusion of spheres below $50 \mu \mathrm{m}$ (which can be cell aggregates). 
RT-PCR. Spheres were collected in $5 \mathrm{ml}$ tubes, centrifuged and resuspended in $4 \mathrm{ml}$ of $0.25 \%$ Trypsin, $2.21 \mathrm{mM}$ EDTA in HBSS (Cellgro, \#25-053-Cl). After 30 min. incubation at $37^{\circ} \mathrm{C}$ cells were centrifuged and resuspendend in Trizol reagent (Life Technologies, \#15596-026). Samples were processed accordingly to manufacturer instructions. Reverse transcription was performed with Cloned AMV First-Strand cDNA Synthesis Kit (Life Technologies, \#12328-040). qPCR was performed with Fast SYBR Green Master Mix (Life Technologies, \#4385612), using primers:

\title{
Mouse Pouf51 (OCT3/4) \\ forward primer 5'-GGAGGGATGGCATACTGTGG-3' \\ reverse primer 5'-ACCTTTCCAAAGAGAACGCC-3'
}

\author{
Mouse Nanog \\ forward primer 5'-TCGAATTCTGGGAACGCCTC-3' \\ reverse primer 5'-CAGGTCTTAACCTGCTTATAGCTCA-3'
}

\author{
Mouse Sox2 \\ forward primer 5'-GGAGGAGAGCGCCTGTTTTT-3' \\ reverse primer 5'-CTGGCGGAGAATAGTTGGGG-3'
}

\author{
Mouse GAPDH \\ forward primer 5'-ATGACATCAAGAAGGTGGTG-3' \\ reverse primer 5'-CATACCAGGAAATGAGCTTG-3'
}

\section{Human biopsies and Immunohistochemistry}

A total of 23 archival biopsy samples from patients with colon adenocracinoma, clear cell kidney carcinoma, and breast ductal carcinoma were obtained from the Louisiana Cancer Research Center (LCRC) tissue biorepository. All samples were de-identified.

Immunohistochemistry was performed using avidin-biotin-peroxidase (Vector Laboratories, Burlingame, California). Briefly, our protocol includes deparaffination in xylene, rehydration through descending grades of alcohol up to water, non-enzymatic antigen retrieval in Citrate buffer, $\mathrm{pH} 6.0$ for 30 minutes at $95^{\circ} \mathrm{C}$, and endogenous peroxidase quenching with $\mathrm{H}_{2} \mathrm{O}_{2}$ in Methanol for 20 minutes. After PBS wash, samples were blocked with 5\% normal goat serum in $0.1 \%$ PBS/BSA. Primary antibodies included mouse monoclonal antibodies against HADHA (1:500 dilution; Abcam), CD66b (1:100 dilution; LS-B7134, Lifespan Biosciences), CPT1 (1:200 dilution; 8F6AE9, Abcam), and HLA-DR (1:100 dilution; L243, Santa Cruz Biotechnology). After PBS washing, sections were incubated with a biotinylated anti-rabbit secondary IgG for 20 minutes, incubated with avidin-biotin-peroxidase (ABC) complexes, and developed with diaminobenzidine (Sigma). All sections were counterstained with Hematoxylin, dehydrated in alcohol, cleared in xylene, and mounted with Permount (Fisher). 


\section{Multi-Labeling and Confocal microscopy}

For immunofluorescence double labeling of paraffin-embedded sections, deparaffinization, antigen retrieval, and blocking were performed as described above (endogenous peroxidase was omitted). Sections were then incubated with a first primary antibody overnight at room temperature and rinsed in PBS. A fluorescein-conjugated secondary antibody was incubated for 1 hour in the dark. After washing with PBS, a second primary antibody was incubated overnight in the dark, followed by rinsing with PBS and incubation with a second rhodamine-tagged secondary antibody for 1 hour. Sections were washed in PBS, mounted in aqueous mounting media with DAPI (Vector Laboratories), and visualized in a confocal microscope (Olympus FV1000).

\section{Statistical analysis}

Data were analyzed by either Student's $t$ test or one-way analysis of variance (ANOVA) followed by Tukey post-test using Graph-Pad Prism analysis software. Results were expressed as mean \pm SEM, and a $P$ values $<0.05$ was considered to be statistically significant.

\section{Results}

\section{Tumor microenvironment induces fatty acid oxidation in T-MDSCs}

The harsh tumor microenvironment created in part by the increased metabolic rate of tumor cells can result in a significant metabolic stress on other cells including T-MDSCs. We aimed to determine the metabolic characteristics of T-MDSCs and establish whether they could regulate the immunosuppressive functions of these cells. To identify the metabolic characteristics of T-MDSCs, a single-cell suspension of s.c. 3LL tumor was simultaneously labeled with the fluorescent glucose analog 2NBDG, and cell-surface markers for MDSCs,

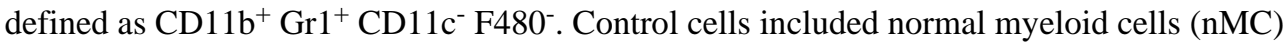
from spleens of naïve mice and MDSCs from spleens of tumor-bearing mice (splenic MDSC) (Fig. 1A and Suppl. Fig. 1A). The non-myeloid fraction containing mostly tumor cells had a significantly increased 2NBDG uptake (Fig. 1A $4^{\text {th }}$ panel and Suppl. Fig 1A), while that in nMCs, splenic MDSCs, and T-MDSCs were all lower. Instead T-MDSCs (but not nMCs or splenic MDSCs) had a high fatty acid uptake, which was similar in the $\mathrm{Ly}^{+} \mathrm{C}^{+}$ monocytic MDSCs (M-MDSC) and the $\mathrm{Ly}_{6 \mathrm{G}}^{+}$granulocytic MDSCs (G-MDSC) subpopulations (Fig. 1B). T-MDSCs had an increased metabolic activity overall as shown by the increased oxygen consumption rate (OCR) and extracellular acidification rate (ECAR) reflecting an increased FAO and glycolysis (Fig 1C). However, the ratio of OCR/ ECAR clearly demonstrated a preferential increase in OCR which confirmed the metabolic reprograming towards fatty acid oxidation (Fig 1D). In addition, the increased OCR in TMDSCs, was accompanied by an increase in mitochondrial biogenesis as shown by higher staining with Mitotracker and enhanced production of superoxide (Mitosox) and ROS (DCFDA) (Fig. 1E and Suppl. Fig. 1B-D). Moreover, T-MDSCs displayed a significantly elevated expression of genes associated with FAO, including CPT1, ACADM, PGC1 $\beta$, and HADHA (Fig. 1F). 
The metabolic reprograming was paralleled by an upregulation of arginase I, elevated production of NO and PNT (Fig. 1G-I, Suppl. Fig. 1E), and increased ability to inhibit Tcell proliferation (Fig. 1J). In addition, T-MDSCs produced higher levels of G-CSF, GMCSF, IL1 $\beta$, IL6, and IL10, cytokines known to promote and sustain MDSC development, however, they also produced higher levels of IL12 (Suppl. Fig. 1F).

\section{Effect of FAO Inhibition on MDSC Function}

We then explored the effect of FAO inhibition on the induction and function of MDSCs using bone marrow-derived MDSCs (BM-MDSC) from normal C57BL/6 mice. BM precursors were activated with G-CSF, GM-CSF, and IL13 and incubated with or without etomoxir, a specific inhibitor of CPT1, which is the first and rate-limiting enzyme in the FAO cycle. Incubation of BM-MDSCs with etomoxir lowered CPT1 enzymatic activity (Suppl. Fig. 2A), but did not alter the proportion of G-MDSCs or M-MDSCs, and did not induce apoptosis or block proliferation of BM-MDSCs (Suppl. Fig. 2B-D). Etomoxir, however, decreased the basal and maximal OCR in BM-MDSCs (Fig. 2A), diminished fatty acid uptake (Fig. 2B), and decreased ATP levels by approximately 40-50\% (Fig. 2C and Suppl. Fig. 2E). Furthermore, etomoxir-treated BM-MDSCs had a significantly decreased ability to block T-cell proliferation (Fig. 2D) and had a lower expression and activity of arginase I (Fig. 2E-F). NO levels were not detected in BM-MDSCs generated in vitro, and PNT levels remained unchanged (data not shown).

We next tested the effect of FAO inhibition in vivo on the accumulation, metabolism, and function of T-MDSCs. The initial experiments tested the effect of daily i.p. injections of etomoxir $(50 \mathrm{mg} / \mathrm{Kg})$ into C57BL/6 mice bearing s.c. 3LL tumors, starting on day 1 after tumor injection and up to day 20, at which time tumors were harvested. Mice did not show any overt toxicity at this dose. Etomoxir treatment decreased the enzymatic activity of CPT1 in T-MDSCs in vivo (Suppl. Fig. 3A), but did not alter the percentage of total T-MDSCs or that of G-MDSC and M-MDSC subsets infiltrating the 3LL tumors (Fig. 3A) or significantly increase their apoptosis. Similar data were found in MCA-38 colon carcinoma (data not shown). However, treatment with etomoxir significantly inhibited fatty acid uptake (Fig. 3B) and ATP production (Fig. 3C) and decreased OCR and ECAR (Fig. 3D). This suggested that inhibition of FAO decreased the overall metabolic activity of T-MDSCs, and that T-MDSCs appeared to be unable to compensate by increasing glycolytic stress response. More importantly, FAO inhibition in vivo decreased the immunosuppressive function of TMDSCs, as demonstrated by their inability to block T-cell proliferation and IFN $\gamma$ production (Fig. 3E). This correlated with a lower expression and production of arginase I, ROS, NO, and PNT (Fig. 3F-I and Suppl. Fig. 3B). In addition, T-MDSCs from etomoxir-treated mice produced significantly lower levels of cytokines critical to the induction and differentiation of MDSCs, such as G-CSF, GM-CSF, IL6, and IL10 (Fig. 3J). In contrast, the levels of IL1 $\beta$ and IL12 remained unchanged. Treatment with etomoxir also diminished the accumulation of $\mathrm{CD}^{+}{ }^{+} \mathrm{FoxP}^{+}$Tregs in the spleens of mice (Suppl. Fig. 3C); minimal numbers of Tregs were found infiltrating $3 \mathrm{LL}$ tumors. Cumulatively, these results suggest that inhibiting FAO blocks the immunosuppressive mechanisms and function of T-MDSCs. Genetic confirmation of the effect of CPT1 inhibition could not be done at present because CPT1 
knock-out mice are embryonic lethal and conditional CPT1 knock-outs are not yet available for testing.

\section{Antitumor effect of FAO inhibition}

FAO inhibitors are used to treat severe coronary disease. Therefore, we tested the effect of FAO inhibitors, administered in different regimens in mice bearing s.c. 3LL lung carcinoma or MCA-38 colon carcinoma. Mice that started etomoxir treatment alone 1 day after tumor implantation and up to day 20 had a significant delay in tumor growth, compared to controls (Fig. 4A-B). Histologic studies showed that tumors from control mice had areas of necrosis, while tumors from etomoxir-treated mice had minimal necrosis, but were infiltrated by mononuclear cells (Fig. 4C, upper panel). Tunel assay showed that tumors from etomoxirtreated mice had a higher number of cells undergoing apoptosis (Fig. 4C, middle panel). Immunohistochemical staining showed no changes in $\mathrm{CD} 31^{+}$endothelial cells or in the number of blood vessels, suggesting that etomoxir did not affect angiogenesis (Fig. 4C, lower panel). We also tested the effect of a second FAO inhibitor, ranolazine, that specifically blocks the trifunctional enzyme HADHA, which catalyzes the last three steps of the FAO cycle (26). Mice treated with ranolazine had a similar antitumor effect as did etomoxir (Fig. 4D). Clonogenic assays showed that FAO inhibition with etomoxir or ranolazine did not inhibit the in vitro growth of 3LL or MCA-38 cells (Suppl. Fig. 4A); etomoxir treatment did not change the number of cancer stem cells (stem cell spheres) or the expression of cancer stem cells markers Sox2, Nanog or Oct3/4 (Suppl. Fig 4B-D). However, the depletion of $\mathrm{CD} 4^{+}$or $\mathrm{CD} 8^{+} \mathrm{T}$ cells in etomoxir-treated mice virtually abrogated the antitumor effect of etomoxir (Fig. 4E and Suppl. Fig. 5A), suggesting that the antitumor effect was at least in part mediated by $\mathrm{T}$ cells. We also tested the possibility that etomoxir would augment T-cell function; however, in vitro cultures of T cells with etomoxir failed to show changes in proliferation, cytokine production, or cytotoxic function (or cytotoxic proteins) (Suppl. Fig. 5B-D).

\section{Effects of FAO inhibition plus low-dose chemotherapy or adoptive cellular therapy (ACT)}

T-MDSCs have been shown to decrease the antitumor effects of chemotherapy and radiation therapy (27) and impair the therapeutic effect of various forms of immunotherapy (1).

Therefore, we tested whether FAO inhibition modulated the antitumor effects of low-dose chemotherapy or ACT. In the initial model, etomoxir was given only for 6 days after tumor implantation, followed by a single dose of CTX $(200 \mathrm{mg} / \mathrm{Kg})$ on day 7 . Results showed an increased antitumor effect on both 3LL and MCA-38 tumor-bearing mice treated with FAO inhibitors plus chemotherapy (Fig. 5A-B). This antitumor effect was also seen when treating mice with "established" 3LL tumors (6-7 days after tumor implantation), in which etomoxir was started on day 6 post-tumor inoculation and given for 5 days (until day 10), followed by a single dose of CTX (Fig. 5C). A similar effect was seen with ranolazine (Fig. 5D).

Furthermore, the combination of etomoxir and ACT, using OT-1 T cells to treat OVAexpressing 3LL tumors, resulted in a significantly better antitumor effect (Fig. 5E). The increased efficacy of T-cell immunotherapy in etomoxir-treated mice correlated with a higher number of adoptively transferred OT-1 T cells (CD45.1 $\left.{ }^{+}\right)$infiltrating the tumors and increased number of cells producing IFN $\gamma$ (Fig. 5F-G). 


\section{Increased fatty acid uptake and expression of CPT1 and HADHA in peripheral blood and T- MDSCs from cancer patients}

Peripheral blood MDSCs from 23 patients with cancer (breast, renal cell carcinoma, bladder cancer, and colon cancer) were isolated and tested for fatty acid uptake. PMNs from normal donors and from the same patients were used as controls. In accordance with previous reports $(21,28)$, G-MDSCs $\left(\mathrm{CD} 14^{\text {neg }} \mathrm{CD} 33^{+} \mathrm{HLA}^{-D R}{ }^{\text {neg }} \mathrm{CD} 6 \mathrm{~b}^{+}\right)$were increased in the peripheral blood of these patients (Fig. 6A-B). Similar to murine T-MDSCs, human GMDSCs had an increased fatty acid uptake (Fig. 6C), and although M-MDSCs also incorporated fatty acids, they represented $<0.9 \%$ of the total circulating MDSCs (data not shown). We then examined the expression of CPT-1 and HADHA in biopsies from patients with colon adenocarcinoma, renal cell carcinoma, and breast ductal carcinoma.

Immunohistochemistry for CPT-1 demonstrated its presence with a punctate cytoplasmic pattern (consistent with its expression in the mitochondria) in tumors cells and a markedly increased expression shown as a solid pattern in inflammatory cells (Fig. 6D, left Panels). Double labeling with anti-CPT1 (fluorescein) and anti-CD66b (rhodamine) showed that the $\mathrm{CD} 6 \mathrm{~b}^{+} \mathrm{T}$-MDSCs expressed significantly higher levels of CPT1, compared to tumor cells (Fig. 6D, right "merge" panels). Similarly, immunohistochemistry for HADHA showed the expression of the enzyme in both tumor cells and inflammatory cells in the stroma (Fig. 6E, left panel). Confocal microscopy following double labeling with anti-HADHA (fluorescein) and anti-CD66b (rhodamine) showed that all CD66b ${ }^{+}$MDSCs were also HADHA ${ }^{+}$(Fig. 6E, right panels). These results suggest that human T-MDSCs have similar metabolic characteristics as murine T-MDSCs. The clinical and biologic significance of these results in patients with cancer is yet to be determined. However, the results support the possibility of testing FAO as an adjuvant to various cancer therapies.

\section{Discussion}

MDSCs are chronic inflammatory cells that inhibit T-cell function and play an important role in promoting tumor growth and facilitating the progression of chronic infections $(4,29)$. Thus, inhibiting MDSCs has been pursued as a means of enhancing the effect of cancer therapies, in particular immunotherapies. However, MDSCs have multiple immunosuppressive mechanisms, which they upregulate depending on the signals encountered in the microenvironment of different tumors. MDSCs in renal cell carcinoma and colorectal carcinoma primarily produce arginase I, while MDSCs in melanoma produce NO and PNT $(21,30,31)$. Targeted inhibition of any of these pathways has not resulted in a significant therapeutic effect, limiting the anti-MDSC therapies to chemotherapeutic agents that suppress their production in the bone marrow. We therefore chose to study the metabolic characteristics of MDSCs and test whether inhibition of these pathways could have a therapeutic application.

In recent years, targeting the energy metabolism pathways of tumors and immune cells has gained interest because of its potential to uncover novel prevention or therapeutic targets $(32,33)$. Thus, we aimed to characterize the energy metabolism pathway(s) of T-MDSCs. The data presented here showed that highly immunosuppressive T-MDSCs activate FAO. Inhibition of FAO using agents approved for the treatment of coronary disease further 
demonstrated the importance of this energy production pathway on the immunosuppressive functions of MDSCs. The molecular mechanisms linking FAO with the regulatory mechanisms in MDSCs are currently unknown; however, the antitumor effect in vivo supports the possibility of testing this therapeutic approach in patients. The mechanisms by which FAO inhibition can cause an antitumor effect are several. Our data strongly suggest that FAO inhibition blocks the immunosuppressive function of T-MDSCs and thus allow T cells to kill tumor cells. However, it is also possible that FAO inhibition also decreases Treg function as proposed by Michalek and colleagues (34). In addition, recent reports have shown that some tumors including pancreatic cancer stem cells and certain myeloid leukemias rely on mitochondrial function for survival $(35,36)$. Although our data failed to demonstrate an inhibition of cancer stem cells in these two murine tumor models, it is still possible that FAO inhibition can affect multiple cells that support the tumor microenvironment. Our data also suggest that, in addition to blocking MDSC function, FAO inhibition could promote antitumor responses since it decreases the production of G-CSF, GM-CSF, and IL6, but does not alter the production of IL12. The net effect of these changes, therefore, appears to be a decrease in the overall immunosuppressive microenvironment in favor of the development of antitumor responses. This is especially significant given the additive effect of etomoxir with CTX, which has been used to modulate the immunosuppressive microenvironment. This combination appears to fully inhibit the immunosuppressive function of T-MDSCs (Suppl. Fig 6).

FAO is also important in other myeloid cell subsets. Elegant studies from several laboratories have shown that M2 macrophages primarily use FAO, while M1 macrophages use glycolysis (18-20). The pathophysiologic importance of this observation was recently demonstrated by Huang and colleagues, showing that inhibiting lipolysis by orlistat decreased the ability of M2 macrophages to control parasitic infection (37). In addition, Herber and colleagues recently showed that $\mathrm{CD} 11 \mathrm{c}^{+}$dendritic cells infiltrating tumors incorporate oxidized lipids that block antigen processing in lysozomes and their assembly on MHC class II, therefore preventing effective antigen presentation and T-cell stimulation (12). This process was inhibited by 5-(tetradecycloxy)-2-furoic acid (TOFA), an inhibitor of fatty acid synthesis. Another important myeloid subpopulation comprises granulocytes, which primarily use glycolysis as a source of ATP (38). Our data show that T-MDSCs, but not splenic MDSCs or nMCs, clearly upregulated FAO, suggesting that factors and/or signals in the tumor microenvironment are responsible for this metabolic reprogramming. In many cancer patients, G-MDSCs are significantly increased in peripheral blood and tumors $(28,39)$, and as shown here, human G-MDSCs have an increased fatty acid uptake and expression of FAO cycle enzymes CPT1 and HADHA.

The factor(s) responsible for inducing FAO in the tumor microenvironment are still unknown. Tumor-derived extracts trigger an increased synthesis of fatty acids in dendritic cells (12). In addition, signaling through Stat6 and IL4, which promote MDSC differentiation, can also induce PGC1 $\beta$ and activate FAO (20). Our data using in vitroderived BM-MDSCs suggest that G-CSF or GM-CSF may also induce FAO (data not shown). A previous study showed that GM-CSF and IL6, instead, upregulate AMPK and glycolysis in BM-MDSCs (40). Recent reports suggest that lactic acid present in high 
concentrations in the tumor microenvironment can activate MDSCs $(29,41)$; however, its role in inducing FAO is unknown. Thus, additional studies are needed to determine the mechanisms causing the metabolic shift towards FAO in T-MDSCs.

How FAO inhibition blocks MDSC immunosuppressive functions is not fully understood, especially given the fact that etomoxir and ranolazine target different enzymes in this pathway. Etomoxir is a non-reversible inhibitor of CPT-1, the enzyme responsible for the initial step of internalization of fatty acids into the mitochondria. Ranolazine is a piperazine derivative that inhibits HADHA, a trifunctional enzyme that catalyzes the last three steps in FAO (26). Both drugs block fatty acid uptake, FAO, and ATP production. More importantly, however, the results demonstrated a novel and potentially important adjuvant effect when combining FAO inhibition with chemotherapy and/or immunotherapy. Phase I clinical trials with etomoxir for the treatment of coronary disease showed toxicities characterized by moderate increases in liver enzymes with chronic use (42). Ranolazine, however, is approved for the treatment of unstable angina. Other FAO inhibitors such as perhexiline (a CPT1 inhibitor) and trimetazidine (an HADHA inhibitor) or lipase inhibitors such as orlistat may, in the present context, inhibit MDSCs. This could be an important development since the current therapeutic approaches aimed at blocking MDSCs rely primarily on the use of chemotherapeutic agents such as gemcitabine (43) and 5-fluorouracil (44) that suppress the bone marrow or the tyrosine kinase inhibitor sunitinib (45). Additionally, certain chemotherapeutic agents increase rather than decrease the accumulation of MDSCs and promote the expression of inhibitory pathways (46). Other approaches have included the use of all-trans-retinoic acid (ATRA) in an attempt to differentiate MDSCs into mature granulocytes (47). Thus, targeting the energy metabolic pathways in T-MDSCs may provide a broader range of effects by globally inhibiting several immunosuppressive mechanisms in T-MDSCs, without causing bone marrow suppression, and allowing $\mathrm{T}$ cells to develop an antitumor function.

Results presented here support the possibility of testing approved FAO inhibitors in the context of chemotherapy or immunotherapy of cancer. It also highlights the importance of studying the metabolism of immune cells in diseases in which MDSC function may be modulated by metabolic manipulation (11, 12), including other malignancies (4), trauma and/or sepsis (48), and chronic infections such as HIV, leishmaniasis and tuberculosis (49).

\section{Supplementary Material}

Refer to Web version on PubMed Central for supplementary material.

\section{Acknowledgments}

Funding: This study has been funded in part by RO1 AI112402, R01CA082689, R01CA107974, P20GM2013501 to ACO, partial support from LA CaTS Center (U54GM104940) to AAA and ACO.

\section{Reference List}

1. Coussens LM, Zitvogel L, Palucka AK. Neutralizing tumor-promoting chronic inflammation: a magic bullet? Science. 2013; 339:286-291. [PubMed: 23329041] 
2. Emens LA, Silverstein SC, Khleif S, Marincola FM, Galon J. Toward integrative cancer immunotherapy: targeting the tumor microenvironment. J Transl Med. 2012; 1070.

3. Mundy-Bosse BL, Lesinski GB, Jaime-Ramirez AC, Benninger K, Khan M, Kuppusamy P, et al. Myeloid-derived suppressor cell inhibition of the IFN response in tumor-bearing mice. Cancer Res. 2011; 71:5101-5110. [PubMed: 21680779]

4. Gabrilovich DI, Ostrand-Rosenberg S, Bronte V. Coordinated regulation of myeloid cells by tumours. Nat Rev Immunol. 2012; 12:253-268. [PubMed: 22437938]

5. Rodriguez PC, Ochoa AC. Arginine regulation by myeloid derived suppressor cells and tolerance in cancer: mechanisms and therapeutic perspectives. Immunol Rev. 2008; 222:180-191. [PubMed: 18364002]

6. Gabrilovich DI, Nagaraj S. Myeloid-derived suppressor cells as regulators of the immune system. Nat Rev Immunol. 2009; 9:162-174. [PubMed: 19197294]

7. Movahedi K, Guilliams M, Van den Bossche J, Van den Bergh R, Gysemans C, Beschin A, et al. Identification of discrete tumor-induced myeloid-derived suppressor cell subpopulations with distinct T cell-suppressive activity. Blood. 2008; 111:4233-4244. [PubMed: 18272812]

8. Raber P, Ochoa AC, Rodriguez PC. Metabolism of L-arginine by myeloid-derived suppressor cells in cancer: mechanisms of T cell suppression and therapeutic perspectives. Immunol Invest. 2012; 41:614-634. [PubMed: 23017138]

9. Wesolowski R, Markowitz J, Carson WE III. Myeloid derived suppressor cells - a new therapeutic target in the treatment of cancer. J Immunother Cancer. 2013; 1:10. [PubMed: 24829747]

10. Ko JS, Zea AH, Rini BI, Ireland JL, Elson P, Cohen P, et al. Sunitinib mediates reversal of myeloid-derived suppressor cell accumulation in renal cell carcinoma patients. Clin Cancer Res. 2009; 15:2148-2157. [PubMed: 19276286]

11. Pearce EL, Pearce EJ. Metabolic pathways in immune cell activation and quiescence. Immunity. 2013; 38:633-643. [PubMed: 23601682]

12. Herber DL, Cao W, Nefedova Y, Novitskiy SV, Nagaraj S, Tyurin VA, et al. Lipid accumulation and dendritic cell dysfunction in cancer. Nat Med. 2010; 16:880-886. [PubMed: 20622859]

13. Ramakrishnan R, Tyurin VA, Veglia F, Condamine T, Amoscato A, Mohammadyani D, et al. Oxidized lipids block antigen cross-presentation by dendritic cells in cancer. J Immunol. 2014; 192:2920-2931. [PubMed: 24554775]

14. Everts B, Amiel E, van der Windt GJ, Freitas TC, Chott R, Yarasheski KE, et al. Commitment to glycolysis sustains survival of NO-producing inflammatory dendritic cells. Blood. 2012; 120:1422-1431. [PubMed: 22786879]

15. Pearce EL, Poffenberger MC, Chang CH, Jones RG. Fueling immunity:insights into metabolism and lymphocyte function. Science. 2013; 342:1242454. [PubMed: 24115444]

16. Sukumar M, Liu J, Ji Y, Subramanian M, Crompton JG, Yu Z, et al. Inhibiting glycolytic metabolism enhances CD8+ T cell memory and antitumor function. J Clin Invest. 2013; 123:4479-4488. [PubMed: 24091329]

17. O'Sullivan D, van der Windt GJ, Huang SC, Curtis JD, Chang CH, Buck MD, et al. Memory CD8(+) T cells use cell-intrinsic lipolysis to support the metabolic programming necessary for development. Immunity. 2014; 41:75-88. [PubMed: 25001241]

18. Rodriguez-Prados JC, Traves PG, Cuenca J, Rico D, Aragones J, Martin-Sanz P, et al. Substrate fate in activated macrophages: a comparison between innate, classic, and alternative activation. $\mathrm{J}$ Immunol. 2010; 185:605-614. [PubMed: 20498354]

19. Odegaard JI, Chawla A. Alternative macrophage activation and metabolism. Annu Rev Pathol. 2011; 6:275-297. [PubMed: 21034223]

20. Vats D, Mukundan L, Odegaard JI, Zhang L, Smith KL, Morel CR, et al. Oxidative metabolism and PGC-1beta attenuate macrophage-mediated inflammation. Cell Metab. 2006; 4:13-24. [PubMed: 16814729]

21. Rodriguez PC, Ernstoff MS, Hernandez C, Atkins M, Zabaleta J, Sierra R, et al. Arginase Iproducing myeloid-derived suppressor cells in renal cell carcinoma are a subpopulation of activated granulocytes. Cancer Res. 2009; 69:1553-1560. [PubMed: 19201693]

22. Sierra RA, Thevenot P, Raber PL, Cui Y, Parsons C, Ochoa AC, et al. Rescue of Notch-1 Signaling in Antigen-Specific CD8+ T Cells Overcomes Tumor-Induced T-cell Suppression and 
Enhances Immunotherapy in Cancer. Cancer Immunol Res. 2014; 2:800-811. [PubMed: 24830414]

23. Rodriguez PC, Quiceno DG, Zabaleta J, Ortiz B, Zea AH, Piazuelo MB, et al. Arginase I production in the tumor microenvironment by mature myeloid cells inhibits T-cell receptor expression and antigen-specific T-cell responses. Cancer Res. 2004; 64:5839-5849. [PubMed: 15313928]

24. Highfill SL, Rodriguez PC, Zhou Q, Goetz CA, Koehn BH, Veenstra R, et al. Bone marrow myeloid-derived suppressor cells (MDSCs) inhibit graft-versus-host disease (GVHD) via an arginase-1-dependent mechanism that is up-regulated by interleukin-13. Blood. 2010; 116:57385747. [PubMed: 20807889]

25. Linher-Melville K, Zantinge S, Sanli T, Gerstein H, Tsakiridis T, Singh G. Establishing a relationship between prolactin and altered fatty acid beta-oxidation via carnitine palmitoyl transferase 1 in breast cancer cells. BMC Cancer. 2011; 11:56. [PubMed: 21294903]

26. MacInnes A, Fairman DA, Binding P, Rhodes J, Wyatt MJ, Phelan A, et al. The antianginal agent trimetazidine does not exert its functional benefit via inhibition of mitochondrial long-chain 3ketoacyl coenzyme A thiolase. Circ Res. 2003; 93:e26-e32. [PubMed: 12869391]

27. DeNardo DG, Brennan DJ, Rexhepaj E, Ruffell B, Shiao SL, Madden SF, et al. Leukocyte complexity predicts breast cancer survival and functionally regulates response to chemotherapy. Cancer Discov. 2011; 1:54-67. [PubMed: 22039576]

28. Solito S, Marigo I, Pinton L, Damuzzo V, Mandruzzato S, Bronte V. Myeloid-derived suppressor cell heterogeneity in human cancers. Ann NY Acad Sci. 2014; 1319:47-65. [PubMed: 24965257]

29. Husain Z, Huang Y, Seth P, Sukhatme VP. Tumor-derived lactate modifies antitumor immune response: effect on myeloid-derived suppressor cells and NK cells. J Immunol. 2013; 191:14861495. [PubMed: 23817426]

30. Sikora AG, Gelbard A, Davies MA, Sano D, Ekmekcioglu S, Kwon J, et al. Targeted inhibition of inducible nitric oxide synthase inhibits growth of human melanoma in vivo and synergizes with chemotherapy. Clin Cancer Res. 2010; 16:1834-1844. [PubMed: 20215556]

31. Zea AH, Rodriguez PC, Atkins MB, Hernandez C, Signoretti S, Zabaleta J, et al. Arginaseproducing myeloid suppressor cells in renal cell carcinoma patients: a mechanism of tumor evasion. Cancer Res. 2005; 65:3044-3048. [PubMed: 15833831]

32. Vander Heiden MG, Cantley LC, Thompson CB. Understanding the Warburg effect: the metabolic requirements of cell proliferation. Science. 2009; 324:1029-1033. [PubMed: 19460998]

33. Warburg O. On respiratory impairment in cancer cells. Science. 1956; 124:269-270. [PubMed: 13351639]

34. Michalek RD, Gerriets VA, Jacobs SR, Macintyre AN, MacIver NJ, Mason EF, et al. Cutting edge: distinct glycolytic and lipid oxidative metabolic programs are essential for effector and regulatory CD4+ T cell subsets. J Immunol. 2011; 186:3299-3303. [PubMed: 21317389]

35. Viale A, Pettazzoni P, Lyssiotis CA, Ying H, Sanchez N, Marchesini M, et al. Oncogene ablationresistant pancreatic cancer cells depend on mitochondrial function. Nature. 2014; 514:628-632. [PubMed: 25119024]

36. Lagadinou ED, Sach A, Callahan K, Rossi RM, Neering SJ, Minhajuddin M, et al. BCL-2 inhibition targets oxidative phosphorylation and selectively eradicates quiescent human leukemia stem cells. Cell Stem Cell. 2013; 12:329-341. [PubMed: 23333149]

37. Huang SC, Everts B, Ivanova Y, O'Sullivan D, Nascimento M, Smith AM, et al. Cell-intrinsic lysosomal lipolysis is essential for alternative activation of macrophages. Nat Immunol. 2014; 15:846-855. [PubMed: 25086775]

38. Kominsky DJ, Campbell EL, Colgan SP. Metabolic shifts in immunity and inflammation. J Immunol. 2010; 184:4062-4068. [PubMed: 20368286]

39. Poschke I, Kiessling R. On the armament and appearances of human myeloid-derived suppressor cells Clin Immunol. 2012; 144:250-268.

40. Hammami I, Chen J, Murschel F, Bronte V, De Crescenzo G, Jolicoeur M. Immunosuppressive activity enhances central carbon metabolism and bioenergetics in myeloid-derived suppressor cells in vitro models. BMC Cell Biol. 2012; 13:18. [PubMed: 22762146] 
41. Colegio OR, Chu NQ, Szabo AL, Chu T, Rhebergen AM, Jairam V, et al. Functional polarization of tumour-associated macrophages by tumour-derived lactic acid. Nature. 2014; 513:559-563. [PubMed: 25043024]

42. Vickers AE. Characterization of hepatic mitochondrial injury induced by fatty acid oxidation inhibitors. Toxicol Pathol. 2009; 37:78-88. [PubMed: 19234235]

43. Ishizaki H, Manuel ER, Song GY, Srivastava T, Sun S, Diamond DJ, et al. Modified vaccinia Ankara expressing survivin combined with gemcitabine generates specific antitumor effects in a murine pancreatic carcinoma model. Cancer Immunol Immunother. 2011; 60:99-109. [PubMed: 20960189]

44. Geary SM, Lemke CD, Lubaroff DM, Salem AK. The combination of a low-dose chemotherapeutic agent, 5-fluorouracil, and an adenoviral tumor vaccine has a synergistic benefit on survival in a tumor model system. PLoS. One. 2013; 8:e67904. [PubMed: 23840786]

45. Bose A, Taylor JL, Alber S, Watkins SC, Garcia JA, Rini BIWJ, et al. Sunitinib facilitates the activation and recruitment of therapeutic anti-tumor immunity in concert with specific vaccination. Int J Cancer. 2011; 129:2158-2170. [PubMed: 21170961]

46. Mikyskova R, Indrova M, Pollakova V, Bieblova J, Simova J, Reinis M. Cyclophosphamideinduced myeloid-derived suppressor cell population is immunosuppressive but not identical to myeloid-derived suppressor cells induced by growing TC-1 tumors. J Immunother. 2012; 35:374384. [PubMed: 22576342]

47. Kusmartsev S, Cheng F, Yu B, Nefedova Y, Sotomayor E, Lush R, et al. All-trans-retinoic acid eliminates immature myeloid cells from tumor-bearing mice and improves the effect of vaccination. Cancer Res. 2003; 63:4441-4449. [PubMed: 12907617]

48. Janols H, Bergenfelz C, Allaoui R, Larsson AM, Ryden L, Bjornsson S, et al. A high frequency of MDSCs in sepsis patients, with the granulocytic subtype dominating in gram-positive cases. J Leukoc Biol. 2014; 96:685-693. [PubMed: 24929004]

49. Macatangay BJ, Landay AL, Rinaldo CR. MDSC: a new player in HIV immunopathogenesis. AIDS. 2012; 26:1567-1569. [PubMed: 22810370] 

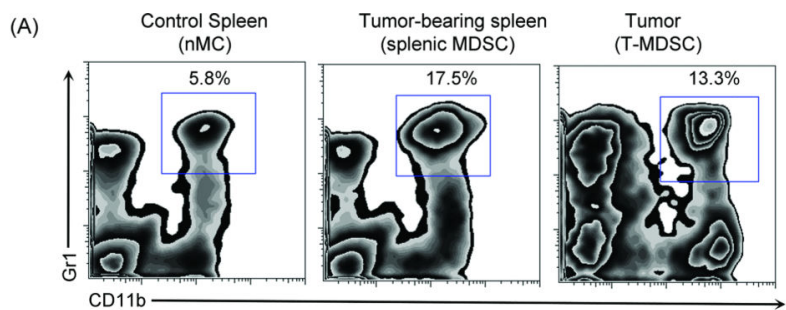

(B)
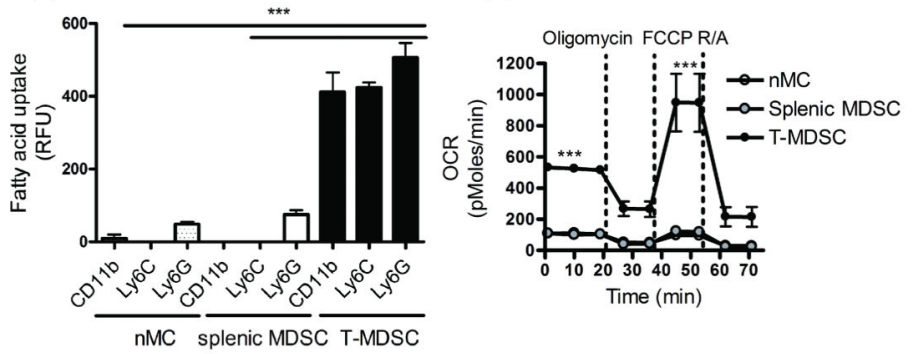

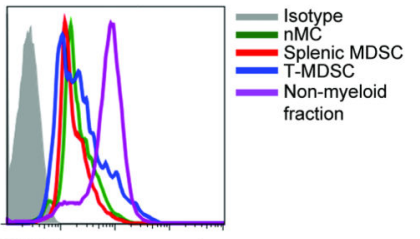

(D)
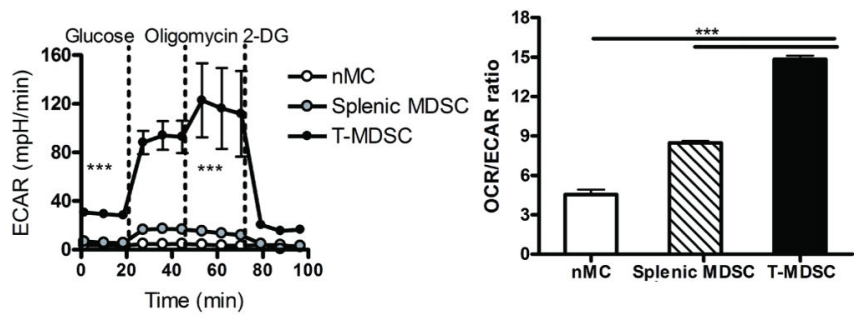

(E)

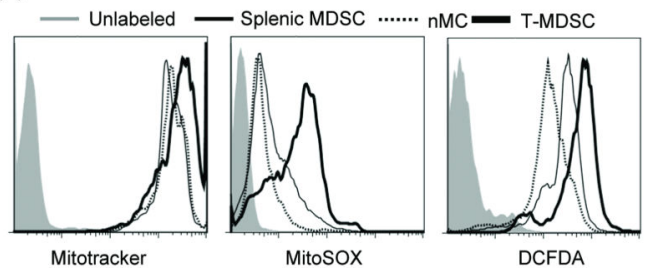

(F)
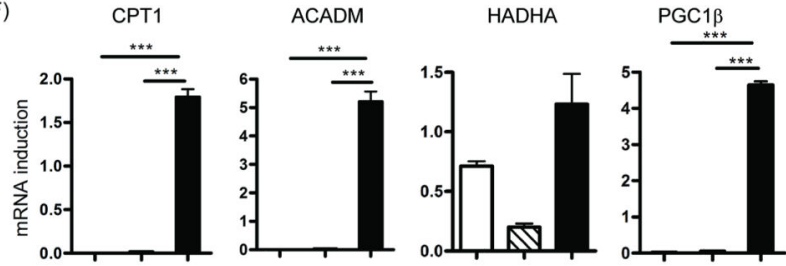

$\square \mathrm{nMC}$ Splenic MDSC T-MDSC
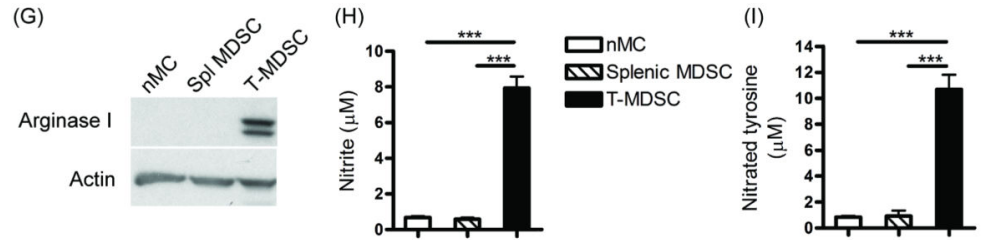

(J)
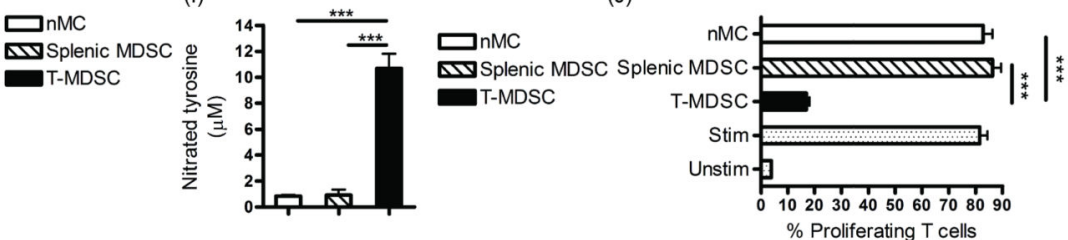

Figure 1. T-MDSCs increase fatty acid uptake, activate FAO, and increase mitochondrial biogenesis and function

(A) 2NBDG incorporation was tested in $\mathrm{CD} 11 \mathrm{~b}^{+} \mathrm{Gr}^{+}$splenocytes from control normal mice (nMC) and from 3LL-bearing mice (splenic MDSC), and 3LL tumor single-cell suspensions (T-MDSC). (B) Fatty acid uptake was measured in CD11b ${ }^{+}, \mathrm{Ly}^{+} \mathrm{C}^{+}$, and $\mathrm{Ly}_{6 \mathrm{G}}^{+}$ populations sorted from control spleens, spleens from 3LL-bearing mice, and 3LL tumor single-cell suspensions. (C) OCR and ECAR were measured under basal conditions and after addition of the indicated drugs. (D) OCR/ECAR ratios. (E) Mitochondrial biogenesis and function was evaluated by assessing mitochondrial mass (Mitotracker), mitochondrial superoxide (Mitosox), and cellular ROS (DCFDA). (F) Quantitative RT-PCR analysis of CPT1, ACADM, PGC1 $\beta$, and HADHA expression. (G-I) Immunosuppressive mechanisms were determined by $(\mathrm{G})$ Western blot for arginase I, $(\mathrm{H})$ NO production as measured by Griess reagent, and (I) PNT production as measured by nitrotyrosine ELISA. (J) Immunosuppressive function as shown by the ability of MDSCs to inhibit the proliferation of $\mathrm{T}$ cells stimulated with anti-CD3/CD28. Data are mean \pm SEM and representative of at least 3 independent experiments. 
(A)

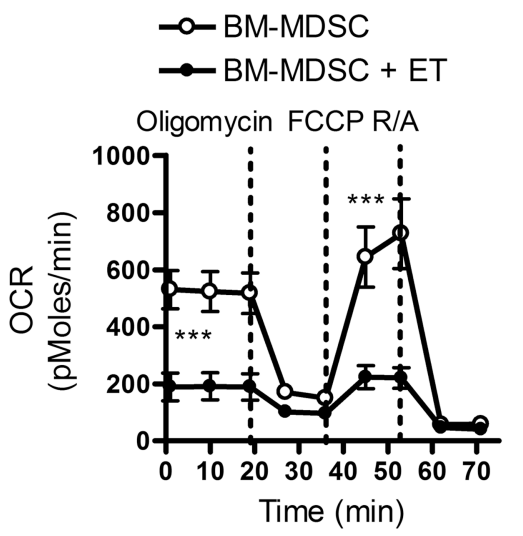

(D)

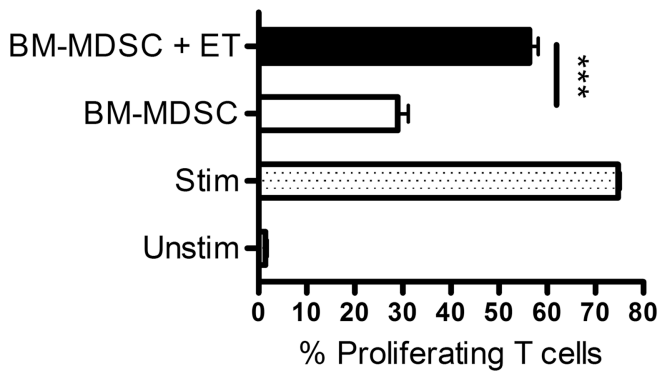

(B)

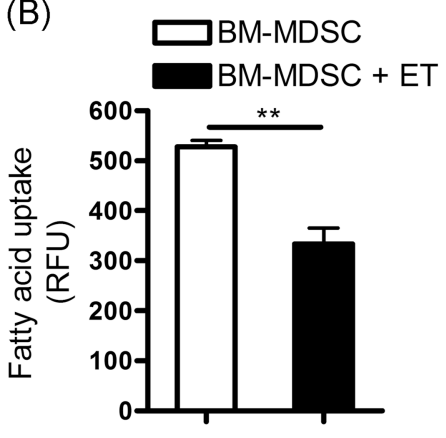

(E)

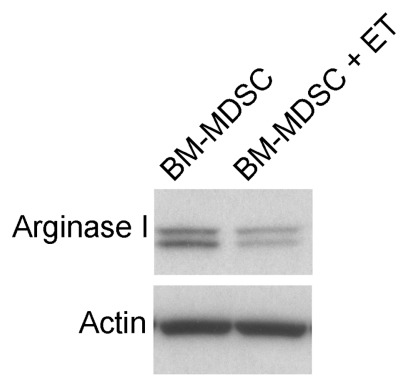

(C)

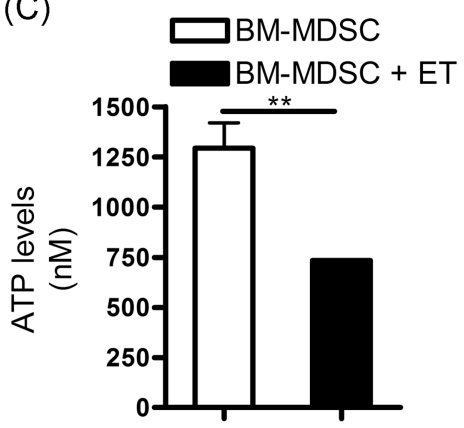

$(\mathrm{F})$

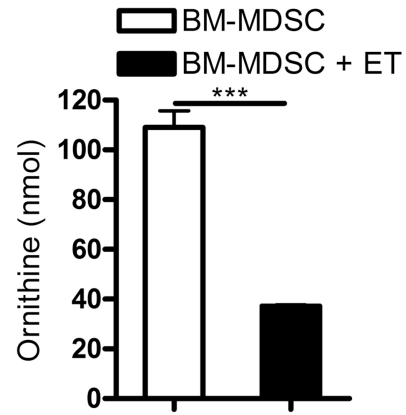

Figure 2. FAO inhibition impairs the function of BM-MDSCs

BM-MDSCs were generated in vitro, as described in Materials and Methods, in the absence or presence of etomoxir $(100 \mu \mathrm{M})$. (A) OCR was measured under basal conditions and after the addition of the indicated mitochondrial regulators. Four days after culture, fatty acid uptake (B), ATP levels (C), immunosuppressive function of BM-MDSCs (D), arginase I expression (E), and arginase I activity (F) were assessed. Data are mean \pm SEM and representative of at least 3 independent experiments. 
(A)

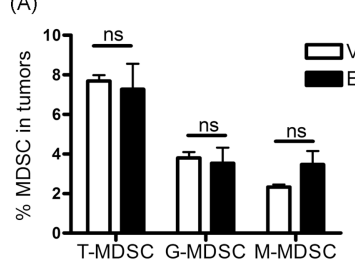

(E)

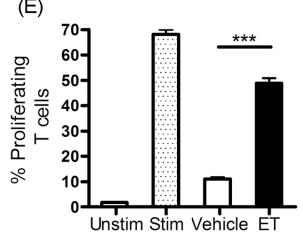

(B)

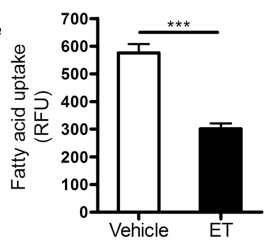

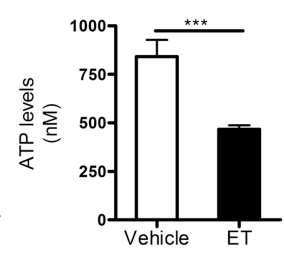

(F)
(D)

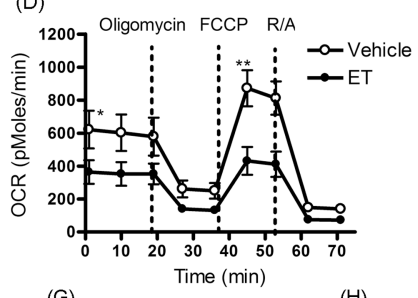

$(\mathrm{G})$
$(\mathrm{H})$

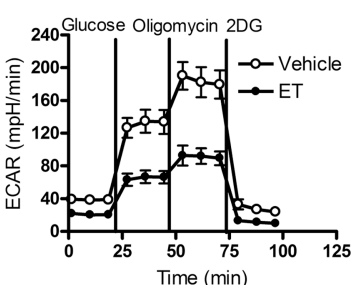

(1)
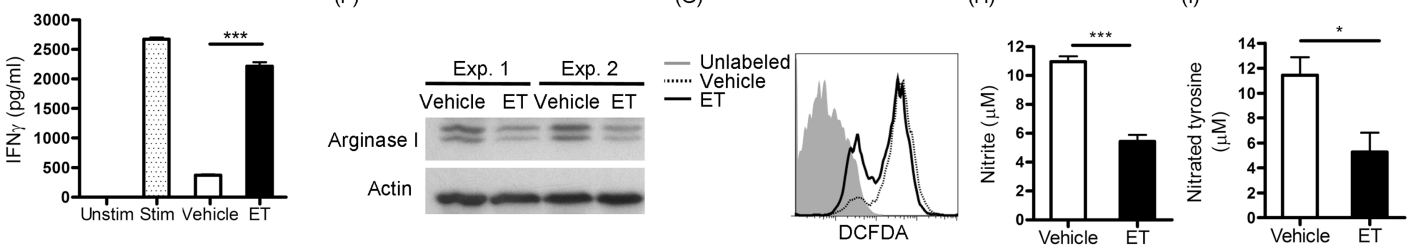

(J)
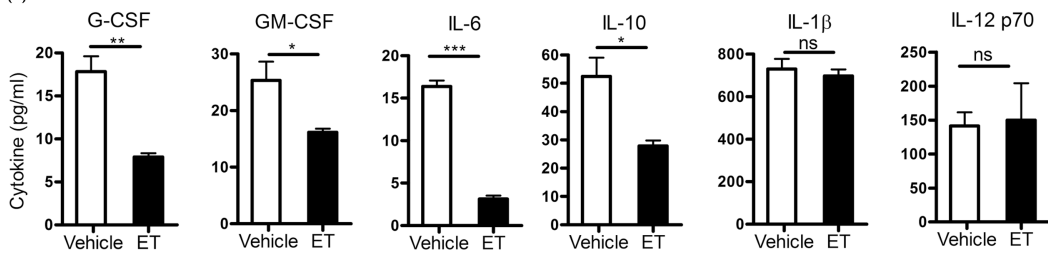

Figure 3. FAO inhibition in vivo decreases fatty acid uptake, ATP production, and immunosuppressive mechanisms in T-MDSCs

C57BL/6 mice bearing s.c. 3LL tumors were treated i.p. with etomoxir $(50 \mathrm{mg} / \mathrm{Kg})$ or PBS for 20 days starting 1 day after tumor injection, and tumors were harvested on day 21. (A) Tumor single-cell suspensions were stained for total T-MDSCs $\left(\mathrm{CD} 11 \mathrm{~b}^{+} \mathrm{Gr} 1^{+}\right)$, G-MDSCs (CD11b ${ }^{+}$Ly6G $^{+}$Ly6C $^{\text {Int }}$ ), and M-MDSCs (CD11b ${ }^{+}$Ly6G $^{\text {lo }}$ Ly6C $^{\text {hi }}$ ). Sorted CD11b ${ }^{+}$GR ${ }^{+}$ cells were tested for fatty acid uptake (B), ATP levels (C), and OCR and ECAR (D). (E) Immunosuppressive function of T-MDSCs was tested by their ability to suppress T-cell proliferation (left) and IFN $\gamma$ production (right). Expression of arginase I (F) and production of ROS (G), NO (H), and PNT (I) were measured in T-MDSCs. (J) Cytokines were measured in T-MDSC lysates using a Bioplex immunoassay. Data are mean \pm SEM and representative of at least 3 independent experiments. 
(A)

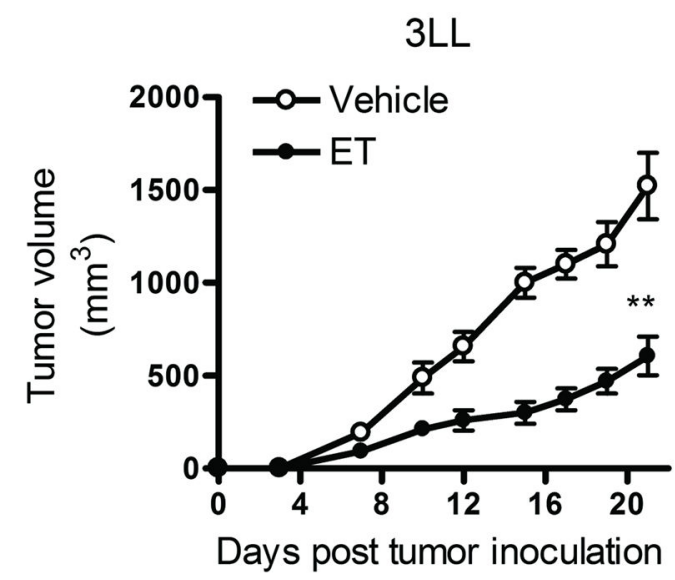

(C)

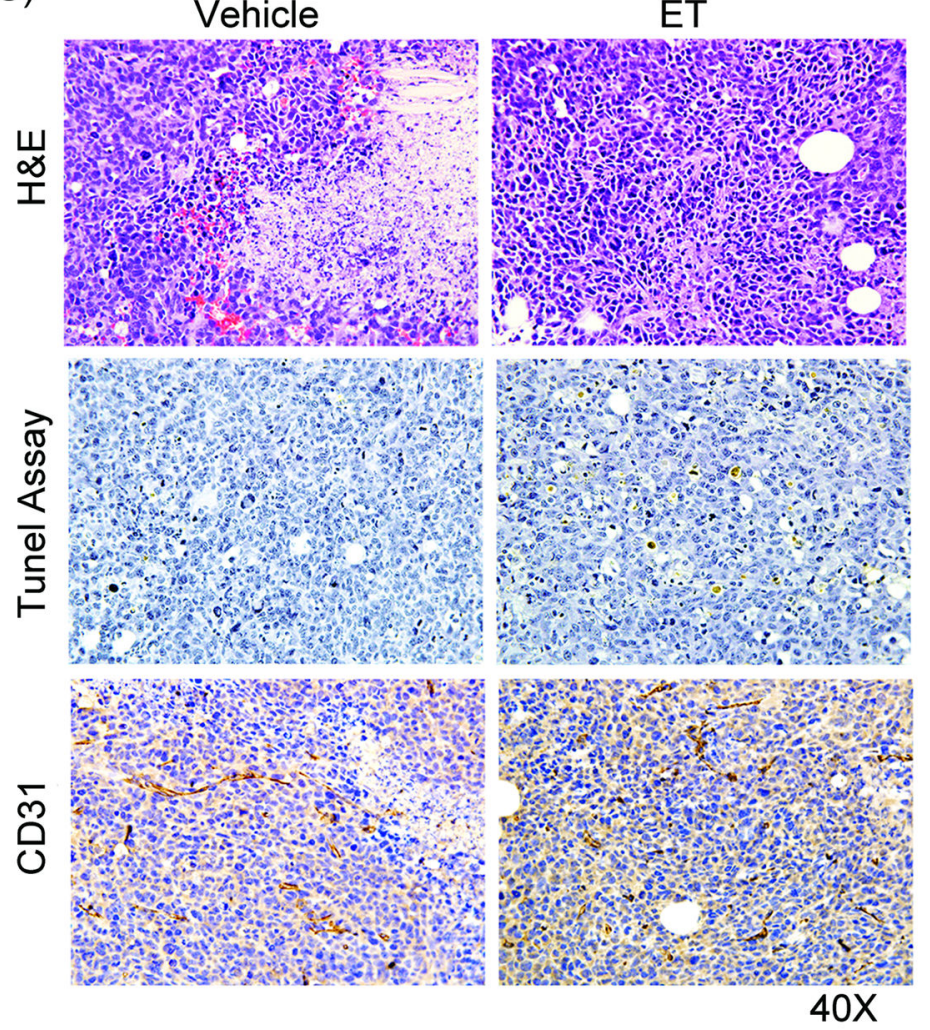

(B) MCA-38

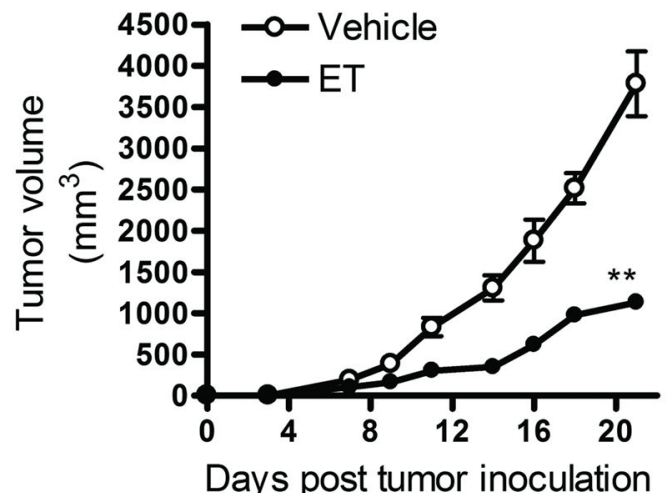

(D)

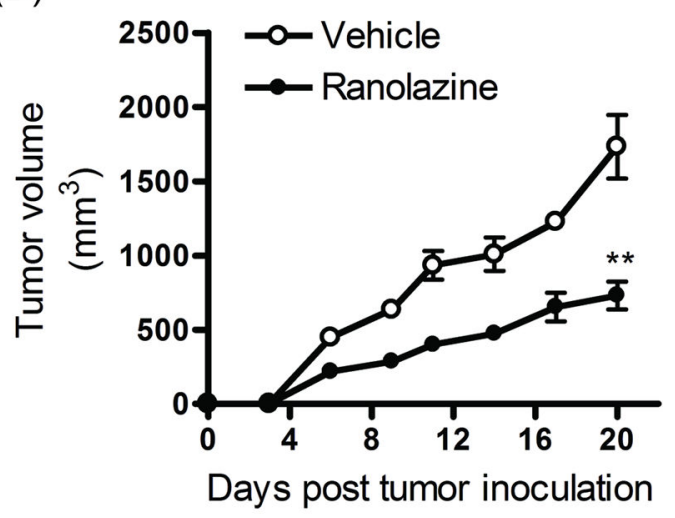

(E) 3LL

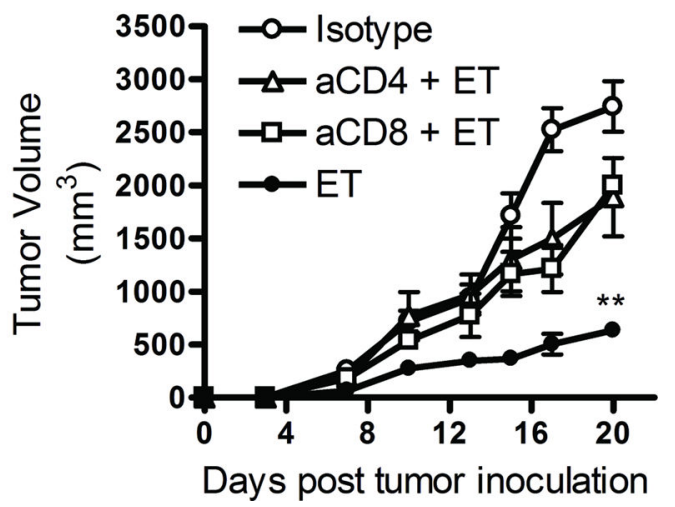

Figure 4. FAO inhibition in vivo significantly delays tumor growth

C57BL/6 mice bearing s.c. 3LL or MCA-38 tumors were treated with $50 \mathrm{mg} / \mathrm{Kg}$ etomoxir i.p. daily for 20 days, and tumors were measured every 2-3 days. (A-B) Tumor growth in control and etomoxir-treated mice (data $=$ mean \pm SEM; $n=5$ mice/group from 3 independent experiments; $\mathrm{p}<0.01$ ). (C) 3LL tumors harvested on day 21 were stained with Hematoxylin \& Eosin, examined for apoptosis by TUNEL assay, and tested for CD31 expression by immunohistochemistry ( $n=3$ mice from 2 independent experiments; $\mathrm{p}<0.01$ ). (D) C57BL/6 mice bearing s.c. 3LL tumors were treated (i.p.) daily for 20 days with 50 
$\mathrm{mg} / \mathrm{Kg}$ of ranolazine (data $=$ mean $\pm \mathrm{SEM} ; \mathrm{n}=5$ mice/group from 3 independent experiments; $\mathrm{p}<0.01$ ). (E) 3LL tumor-bearing mice were treated with etomoxir plus depleting antibodies for $\mathrm{CD} 4$ or $\mathrm{CD} 8$. Controls received IgG isotype (data $=$ mean $\pm \mathrm{SEM} ; \mathrm{n}$ $=5$ mice/group from 2 independent experiments). 
(A)

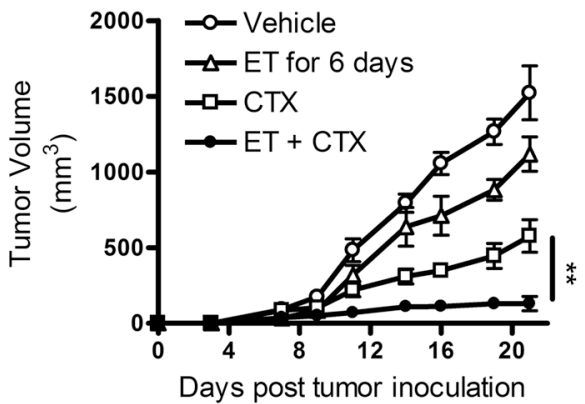

(B)

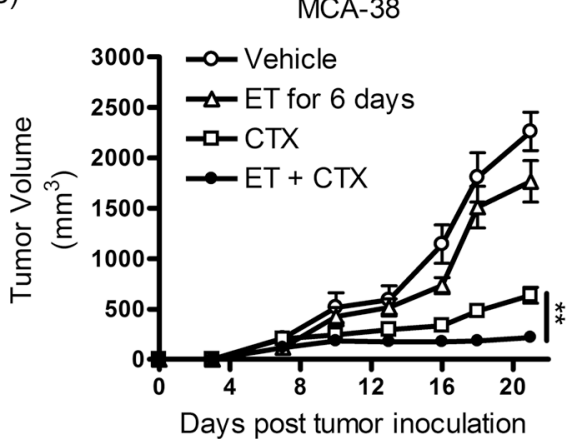

(C)<smiles>[AlH2]</smiles>

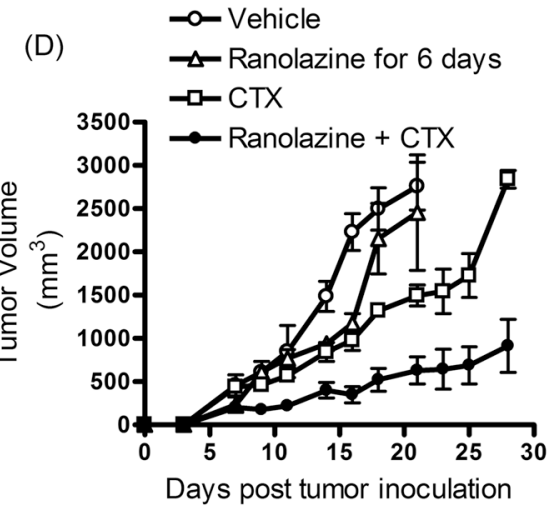

$(\mathrm{F})$

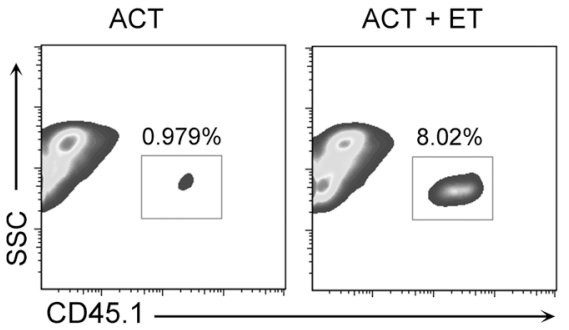

(E)

3LL-OVA

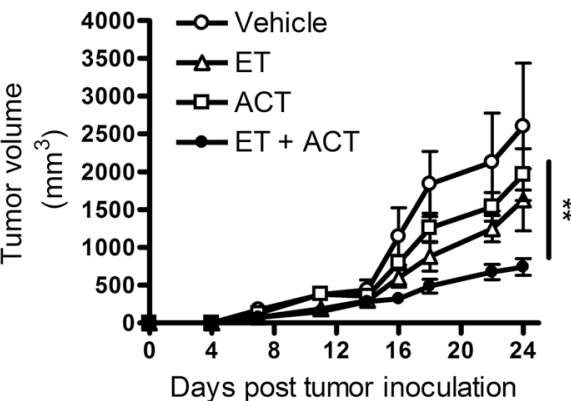

(G)

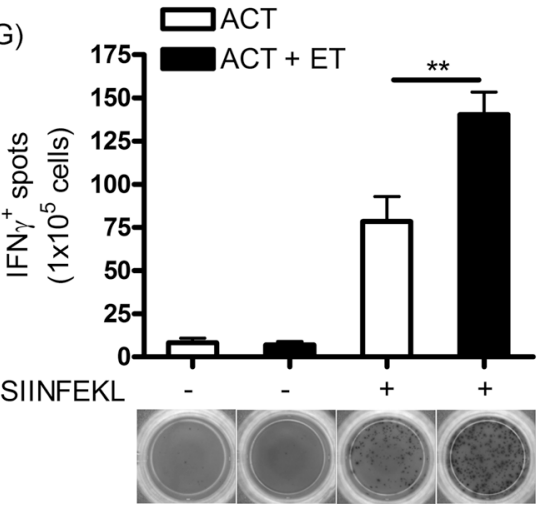

Figure 5. FAO inhibition is synergistic with chemotherapy and ACT

(A-B) C57BL/6 mice bearing s.c. 3LL (A) or MCA-38 (B) tumors were treated with 50 $\mathrm{mg} / \mathrm{Kg}$ etomoxir i.p. for 6 days (starting 1 day after tumor injection) plus a single injection of $200 \mathrm{mg} / \mathrm{Kg}$ CTX on day 7. Tumor growth was followed for 21 days. (C-D) Mice with established tumors were treated with etomoxir (C) or ranolazine (D) i.p. for 5 days (day 610) followed by a single injection of CTX on day 11. (E) For ACT, mice with 3LL-OVA tumors were treated with etomoxir daily starting 1 day after tumor injection. The indicated groups received an i.v. injection of $2.5 \times 10^{6}$ activated OT-1 cells, followed by vaccination with SIINFEKL (100 $\mu \mathrm{g} / \mathrm{mouse}$ ) on days 14 and 15, respectively. Tumor growth was monitored (data for all combination therapies = mean $\pm S E M ; n=5$ mice/group from 2 independent experiments). (F) Tumors were isolated from mice receiving ACT and tested for the numbers of tumor-infiltrating OT-1 cells (CD45.1 $\left.{ }^{+}\right)$. (G) Splenic T cells were 
stimulated ex vivo with SIINFEKL $(1 \mu \mathrm{g} / \mathrm{ml})$, and the frequency of IFN $\gamma$-producing cells was tested by ELISPOT (data $=$ mean \pm SEM; $\mathrm{n}=5$ mice/group from 1 experiment). 


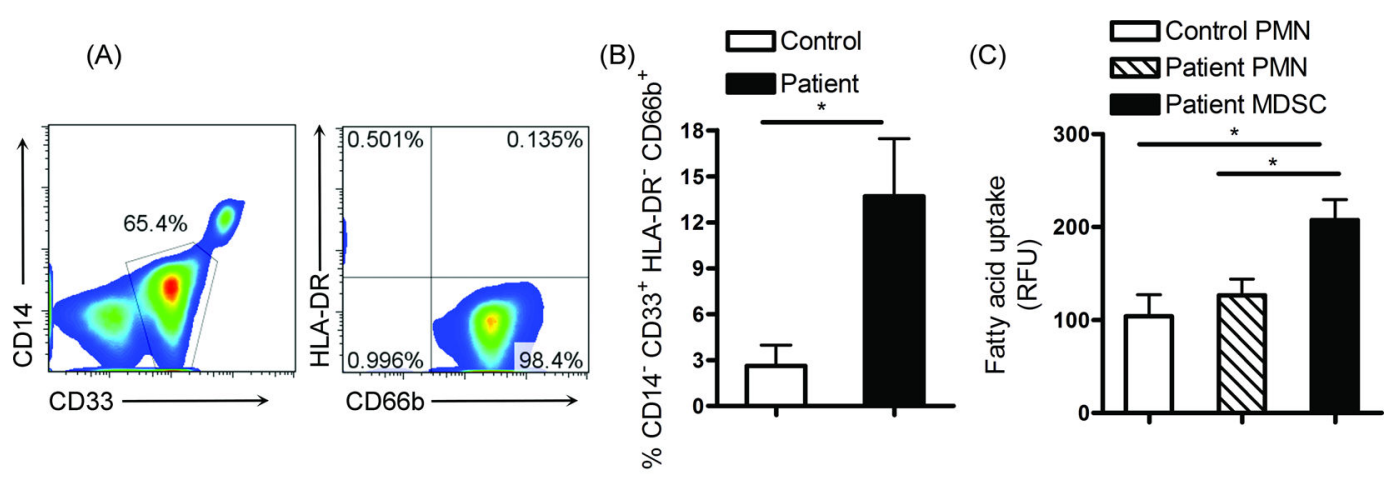

(D)
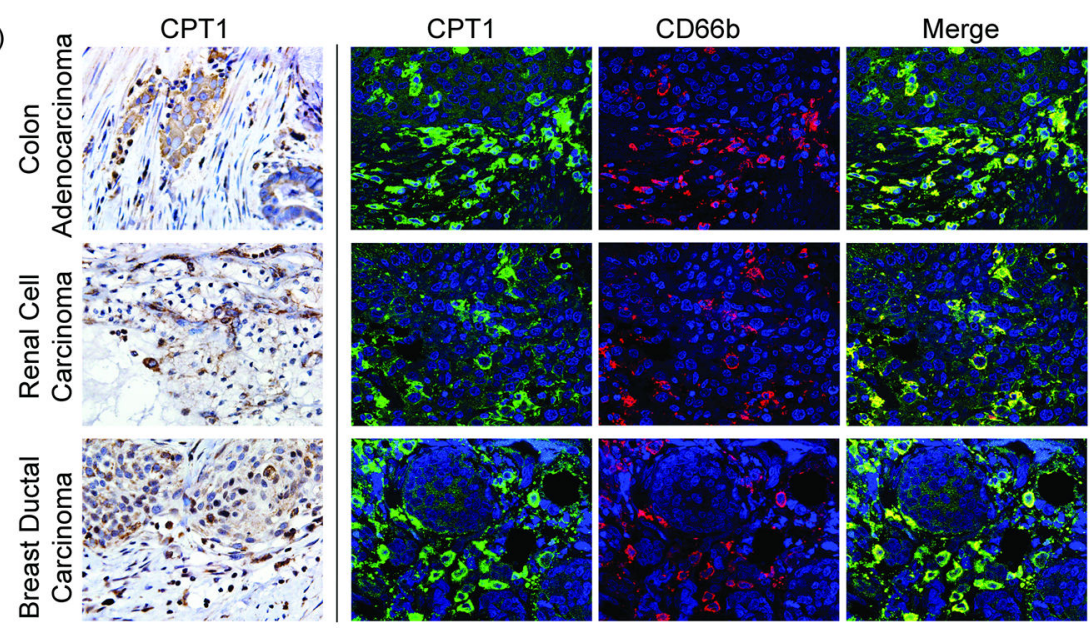

(E)
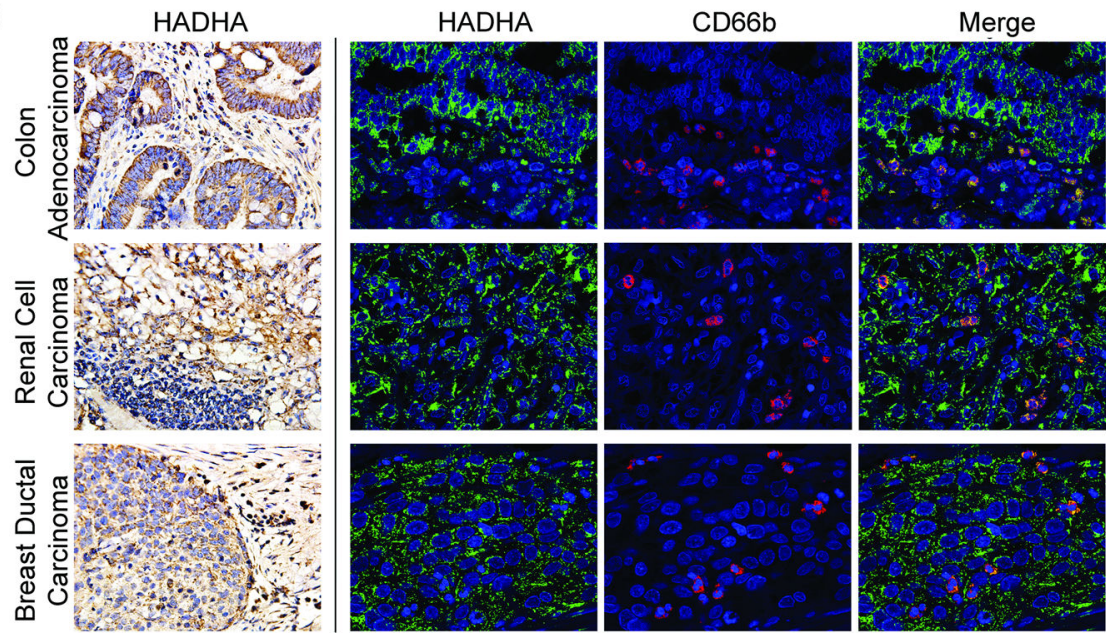

Figure 6. Peripheral blood MDSCs from cancer patients have an increased fatty acid uptake, and T-MDSCs express CPT-1 and HADHA

(A-B) Peripheral blood MDSCs $\left(\mathrm{CD} 14^{\text {neg }} \mathrm{CD} 33^{+} \mathrm{HLA}^{-D R^{\text {neg }}} \mathrm{CD}^{\mathrm{C}} 6 \mathrm{~b}^{+}\right)$from 23 patients with breast, renal cell carcinoma, bladder cancer, and colon cancer were measured by flow cytometry. (C) MDSCs were sorted and tested for fatty acid uptake. Control cells included PMNs from patients and normal controls. (D) Tumor samples from 3 patients with colon carcinoma, renal cell carcinoma, and ductal carcinoma of the breast were tested for CPT1 by immunohistochemistry (left panels) and immunofluorescence double labeling with CPT-1 (fluorescein) and CD66b (Rhodamine). (E) Tissues from (D) were tested for HADHA by 
immunohistochemistry (left panels) and immunofluorescence double labeling with HADHA (fluorescein) and CD66b (Rhodamine). 